\title{
Topoisomerase I as a target of erlotinib and gefitinib: Efficacy of combined treatments with camptothecin
}

\author{
REFAEL PELEG ${ }^{1}$, DMITRI BOBILEV ${ }^{2}$ and ESTHER PRIEL ${ }^{1}$ \\ ${ }^{1}$ The Shraga Segal Department of Microbiology, Immunology and Genetics, \\ Faculty of Health Sciences, Ben-Gurion University of the Negev; \\ ${ }^{2}$ Department of Oncology, The Soroka Hospital Medical Center, Beer Sheva, Israel
}

Received October 3, 2013; Accepted November 13, 2013

DOI: 10.3892/ijo.2014.2244

\begin{abstract}
Topoisomerases are essential nuclear enzymes that work to resolve topological problems that normally occur during DNA metabolism. Their involvement in crucial DNA associated-processes, such as replication, transcription and repair, mark them as a target of chemotherapeutic drugs such as camptothecins (CPTs). Therefore, finding other agents that may alter their activity is of great importance. Previous data showed that certain tyrosine kinase antagonists, tyrphostins, inhibit the catalytic activity of the cellular topoisomerase I (topo I). We examined the effect of clinically used tyrosine kinase inhibitors (TKIs), erlotinib and gefitinib, on topo I in breast and prostate cancer cells. While erlotinib and gefitinib inhibit cellular topo I in treated cells without affecting the levels of the enzyme protein, in vitro assays show that erlotinib, but not gefitinib, inhibits the DNA relaxation activity of purified topo I. Erlotinib was found to reduce the DNA-binding ability of topo I, however, the reduction in topo I activity in gefitinib-treated cells is probably due to post-translational modifications of the enzyme protein. A combined treatment of either erlotinib or gefitinib with CPT increased the effect of CPT on the activity of cellular topo I, which supports the increased anticancer effect observed in MCF7 cells. These results suggest that topo $I$ is a novel target of erlotinib and a combination of TKIs with topo I inhibitors may be an effective treatment for breast cancer.
\end{abstract}

\section{Introduction}

DNA topoisomerases are essential nuclear enzymes that function to resolve topological problems in DNA, which

Correspondence to: Professor Esther Priel, The Shraga Segal Department of Microbiology, Immunology and Genetics, Faculty of Health Sciences, Ben-Gurion University of the Negev, Beer Sheva, Israel

E-mail: priel@bgu.ac.il

Key words: breast cancer, camptothecin, EGFR, erlotinib, gefitinib, topoisomerase I normally occur during replication, transcription, and other DNA-associated processes. The family of topoisomerases has two major members - type I (topo I) and type II (topo II), and their catalytic activity involves the formation of transient covalent bridges of enzyme-DNA complexes (1-5). The involvement of these enzymes in essential cellular processes tagged topoisomerases as important targets for anticancer treatments and for the development of potent, more effective, anticancer drugs.

Topoisomerase I interacts with camptothecin (CPT) and several of its analogs (i.e. topotecan, irinotecan) at the interface of the enzyme-DNA complex to induce cell-death. Topo I is the only target of these alkaloid compounds $(6,7)$. Clinical activity includes phase I-III studies for the indicated FDA approved agents for ovarian, colon, small- and non-small cell lung cancers. Other topoisomerase I inhibitors are being tested in the clinic, in the treatment of pancreatic, breast and hematologic malignancies (6).

Preclinical studies on cell lines and tumor xenografts demonstrated the antitumor activity of EGFR-TK-inhibitors, as single agent or in combination with other drugs, including topoisomerase interacting agents (8-11).

The role of epidermal growth factor receptor (EGFR) has been identified in various tumors $(12,13)$. The EGFR signaling pathway is one of the most important pathways that regulate growth, survival, proliferation, and differentiation in cells.

The EGFR is overexpressed, dysregulated or mutated in many epithelial malignancies, and its activity is important in tumor growth, progression and metastatic ability. This has made these receptors the target of development of anticancer treatment approaches $(13,14)$.

The most predominant clinical approach of EGFR inhibition includes monoclonal antibodies that target the extracellular domain of the receptor, and small molecule tyrosine kinase inhibitors (TKIs) that inhibit the receptor's catalytic activity (15). Erlotinib (Tarceva) and gefitinib (Iressa, ZD-1839) are small-molecule tyrosine kinase inhibitors directed against the epidermal growth factor receptor. These molecules block the intracellular autophosphorylation of the receptor, and affects EGFR-mediated cell proliferation (16).

It was previously demonstrated in our lab, and others, that certain small synthetic tyrosine kinase antagonist molecules, 
tyrphostins, inhibit the catalytic activity of purified topo I enzyme (17), as well as the cellular topo I in drug-treated cells (18).

In recent years, it has been shown in vitro and in vivo, that the combined treatment of human colorectal cancer cells, with anti-EGFR drugs and with topoisomerase I inhibitors increased the antitumor activity of either agent alone $(8,9,19)$ and with no increased toxicity $(10,19)$. Phase II studies have demonstrated that combined therapy of triple negative breast cancer (TNBC) with cetuximab (an anti-EGFR antibody) and cisplatin dramatically increased patients' response rate, compared to cisplatin treatment alone and doubled their progression-free survival duration (20).

Gefitinib was found to modulate SN-38 (the active metabolite of CPT-11) ability to inhibit topo I in colorectal tumor cell lines, to accumulate cells in S-phase, and to induce apoptosis (21). Erlotinib was found to inhibit tumor growth and metastasis in a TNBC xenograft model (20).

These findings indicate that EGFR-TKIs can enhance the antitumor activity of other anticancer agents, as well as topoisomerase inhibitors, without enhanced toxicity, and will be effective against tumor cells.

There is an immense value in finding drugs that can modulate several cellular targets. Therefore, understanding the full potential of these drugs and their mode of action can ensure effective treatment protocols.

As tyrphostins are not clinically approved, we sought to investigate the effect of erlotinib and gefitinib on topo I. In this study we show for the first time that topo I is an additional target of erlotinib and gefitinib in drug-treated tumor cells. Their mechanism differs from camptothecins, known inhibitors of topo I. While erlotinib inhibits topo I by affecting the ability of the enzyme to bind DNA, gefitinib probably affects the enzyme through regulation of the EGFR signaling pathway. Furthermore, combined treatments based on low doses of erlotinib or gefitinib and CPT enhanced the inhibitory effect of CPT in MCF7 cells.

\section{Materials and methods}

Cells. MCF7 and PC3 cell lines were cultured as a monolayer in DMEM or RPMI-1640 medium (Biological Industries Beith Haemek, Israel), respectively, supplemented with $10 \%$ fetal bovine serum, $100 \mathrm{U} / \mathrm{ml}$ penicillin, $100 \mu \mathrm{g} / \mathrm{ml}$ streptomycin and L-glutamine. Cell lines were grown in a humidified incubator supplemented with $5 \% \mathrm{CO}_{2}$, at $37^{\circ} \mathrm{C}$.

Enzymes, antibodies and compounds. Erlotinib (Tarceva $\left.{ }^{\circledR}\right)$ was kindly provided by Roche Diagnostics GmbH Pharma Research, Penzberg, Germany. Gefitinib (Iressa ${ }^{\mathrm{TM}}$ ) was kindly provided by AstraZeneca Pharmaceuticals (Cheshire, UK). Stock solutions of erlotinib and camptothecin (Sigma, Israel), at $20 \mathrm{mM}$ (dissolved in 100\% DMSO), and of gefitinib, at $50 \mathrm{mM}$, were stored in aliquots at $-70^{\circ} \mathrm{C}$ and diluted in DMSO before being added to the reaction mixture or to the cell culture medium. Stock solution of etoposide (Teva, Israel), at $34 \mathrm{mM}$, was stored at room temperature.

Purified calf thymus topoisomerase I was purchased from Takara Bio Inc. Supercoiled DNA plasmid pUC19 and E. coli (cells with a cloned pseT gene of bacteriophage T4) T4 poly- nucleotide kinase were purchased from Fermentas (Hanover, MD, USA).

The primary antisera were as follows: monoclonal mouse anti- $\beta$-actin antibody (MP Biomedicals, LLC); goat polyclonal IgG (C-15) anti-topo I (Santa Cruz Biotechnology Inc., CA, USA).

Appropriate horseradish peroxidase secondary antibodies were purchased from Santa Cruz Biotechnology Inc. Enhanced chemiluminescence (ECL) reagents were purchased from Biological Industries Beith Haemek, Israel.

Topo I DNA-binding sequence oligonucleotides were obtained from Sigma-Aldrich, Israel. $\left[\gamma^{3}{ }^{32}\right.$ P $]$ ATP was purchased from Saifan Precision Instruments Ltd., Israel.

Cell proliferation assay. Cells were plated as triplicate in 96-well plates at a density of 5,000 cells/well in $100 \mu \mathrm{l}$ of DMEM medium and incubated overnight. Various concentrations of the drugs were added for different time intervals. Control cultures received medium containing the highest concentration of the vehicle (DMSO) present in any treatment group. Plates were incubated at $37^{\circ} \mathrm{C}$. Cell cytotoxicity was measured by the Neutral Red assay (22). The $\mathrm{CC}_{50}$ value for each drug was calculated.

Nuclear protein extracts preparation. Nuclear extracts for topoisomerase assays and western blot analysis from MCF7 and PC3 cells was prepared as described $(23,24)$ except that a mixture of protease inhibitors (final concentrations: $2 \mu \mathrm{g} / \mathrm{ml}$ aprotinin, $2 \mu \mathrm{g} / \mathrm{ml}$ leupeptin, $1 \mu \mathrm{g} / \mathrm{ml}$ pepstatin A, $2 \mu \mathrm{g} / \mathrm{ml}$ antipain, $100 \mu \mathrm{g} / \mathrm{ml}$ PMSF) was added to the extraction buffers. Total protein concentration was determined using the Bio-Rad protein assay kit (Bio-Rad Lab, CA, USA).

Topoisomerase I assay. Purified calf thymus topo I (1 U) or $25 \mathrm{ng}$ of total nuclear proteins from drug-treated and untreated cell lines was added to a topo I specific reaction mixture containing, at a final volume of $25 \mu \mathrm{l}: 20 \mathrm{mM}$ Tris- $\mathrm{HCl}$ (pH 8.0), $1 \mathrm{mM}$ dithiothreitol, $20 \mathrm{mM} \mathrm{KCl}, 10 \mathrm{mM}$ $\mathrm{MgCl}_{2}, 1 \mathrm{mM}$ EDTA, $30 \mu \mathrm{g} / \mathrm{ml}$ bovine serum albumin and 225 ng pUC19 supercoiled DNA plasmid. Erlotinib, at various concentrations, was added to the reaction mixture prior to the addition of the enzyme. Following incubation at $37^{\circ} \mathrm{C}$ for $30 \mathrm{~min}$, the reaction was terminated by adding $5 \mu \mathrm{l}$ of stopping buffer [final concentration; $1 \%$ sodium dodecyl sulfate (SDS), $15 \%$ glycerol, $0.5 \%$ bromophenol blue and $50 \mathrm{mM}$ EDTA $\mathrm{pH}$ 8.0]. The reaction products were analyzed by electrophoresis on $1 \%$ agarose gel using a TBE buffer $(89 \mathrm{mM}$ Tris-HCl, $89 \mathrm{mM}$ boric acid, and $62 \mathrm{mM}$ EDTA) at $1 \mathrm{~V} / \mathrm{cm}$, stained by ethidium bromide $(1 \mu \mathrm{g} / \mathrm{ml})$, and photographed using a short wavelength UV lamp (Chemilmager ${ }^{\mathrm{TM}} 5500$ equipment, Alpha Inotech Corp., CA, USA). Densitometric analysis of the results was performed using the EZQuant-Gel software (EZQuant, Rehovot, Israel), and the percentage of topo I inhibition was calculated (17,18).

Determination of the level of topo I protein by western blot analysis. Equal amounts of nuclear proteins derived from MCF7 cells that were subjected to different treatments, were analyzed by western blot analysis as previously described $(24,25)$ using either anti topo I antibodiy (Santa Cruz Biotechnology Inc.), or 
anti- $\beta$-actin antibodies (MP Biomedicals, LLC). The immunocomplexes were detected by enhanced chemiluminescence (ECL).

Electromobility shift assays. In vitro topo I DNA-binding activity was assayed by incubating in a total volume of $25 \mu \mathrm{l}$ for $5 \mathrm{~min}$ at $37^{\circ} \mathrm{C}$. A $\left[\gamma^{-32} \mathrm{P}\right]$-labeled double-stranded oligonucleotide (31-bp) containing the consensus sequence for topo I was added to the reaction mixture. Erlotinib $(100 \mathrm{pM})$ or CPT $(120 \mu \mathrm{M})$ were added to each sample. The reaction products were electrophoresed on a $6 \%$ native polyacrylamide gel that had been pre-electrophoresed for $1 \mathrm{~h}$. Gel was then dried for $30 \mathrm{~min}$ at $80^{\circ} \mathrm{C}$ and authoradiography was performed.

Oligonucleotides. Oligonucleotides sequence: sense 5'-CATG AAAAAAGACTTAGAAAAATTTTTAAAA-3'; antisense 5'-TTTTAAAAATTTTTCTAAGTCTTTTTTCATG-3' (26). Annealing was performed as followed: equal molar concentrations of oligonucleotides were incubated in STE buffer $(10 \mathrm{mM}$ Tris pH 8.0, $50 \mathrm{mM} \mathrm{NaCl}, 1 \mathrm{mM}$ EDTA) in a PCR cycle, at $95^{\circ} \mathrm{C}$ for $2 \mathrm{~min}$, afterwards it was cooled to $25^{\circ} \mathrm{C}$ for $45 \mathrm{~min}$. Products were stored at $4^{\circ} \mathrm{C}$.

Labeling 5'-protruding termini of DNA by exchange reaction. $\mathrm{T} 4$ polynucleotide kinase $(10 \mathrm{U})$ was added to a specific reaction mixture containing, at a final volume of $20 \mu \mathrm{l}: 20 \mathrm{pmol}$ specific oligonucleotides, $0.1 \mathrm{M}$ imidazole- $\mathrm{HCl}(\mathrm{pH} 6.4$ at $25^{\circ} \mathrm{C}$ ), $18 \mathrm{mM} \mathrm{MgCl}_{2}, 5 \mathrm{mM}$ DTT, $0.1 \mathrm{mM}$ spermidine, $0.1 \mathrm{mM}$ EDTA, $0.1 \mathrm{mM}$ ADP, 40 pmol $\left[\gamma^{32}{ }^{32} \mathrm{P}\right] \mathrm{ATP}$ and $4.8 \%(w / v)$ polyethylene glycol 6000 . Following incubation at $37^{\circ} \mathrm{C}$ for $30 \mathrm{~min}$, the reaction was terminated by adding $1 \mu \mathrm{l}$ 0.5 M EDTA (pH 8.0). Labeled DNA was separated using a HiYield Gel/PCR DNA fragment extraction mini-prep kit (RBC Bioscience).

Band depletion assay. The band depletion assay was performed essentially as previously described (10). MCF7 cells $\left(3 \times 10^{6}\right.$ cells/flask) were preincubated with erlotinib for $3 \mathrm{~h}$ prior to the treatment with CPT for $1 \mathrm{~h}$. The cells were removed from the flask by scraping without removing the medium (to prevent reversal of the cleavable complex), followed by centrifugation $\left(1,000 \mathrm{rpm}, 5 \mathrm{~min}, 4^{\circ} \mathrm{C}\right)$. Denaturing buffer ( $2 \% \mathrm{SDS}, 62.5 \mathrm{mM}$ Tris-HCl pH 6.8, and $1 \mathrm{mM}$ EDTA) was immediately added to the cell pellet, and the samples were mixed by vortexing until the turbidity disappeared. Samples were sonicated to diminish viscosity (40 bursts of 2 min each at two-thirds of the maximum output of microtip). Equal volumes of protein samples were analyzed on $7.5 \%$ SDS-PAGE followed by western blot analysis with anti-topo I antibodies.

\section{Results}

Combined treatment of erlotinibor gefitinib with CPT increases the anticancer effect of CPT in MCF7. In order to investigate the mechanism of the combined treatment with topoisomerase- and tyrosine kinase inhibitors, we examined this effect in MCF7 breast and PC3 prostate cancer cell lines. First, cells were exposed to various concentrations $(0-20 \mu \mathrm{M})$ of erlotinib or gefitinb for $24 \mathrm{~h}$. While cells exhibit higher sensitivity to gefitinib treatment $\left(\mathrm{IC}_{50}\right.$ concentrations are $9 \pm 1 \mu \mathrm{M}$ for MCF7 and $16 \pm 1.4 \mu \mathrm{M}$ for PC3), MCF7 and PC3 cells were quite resistant to erlotinib treatment (data not shown), suggesting that erlotinib alone is not efficient as a cytotoxic drug for these cells. Therefore, an acceptable dose of erlotinib was chosen for the evaluation of the combined treatment. Low doses of CPT were also selected for the investigation of the combined treatment on the viability of MCF7 and PC 3 cells. CPT $(0.1$ or $0.02 \mu \mathrm{M})$ and either erlotinib $(0.5 \mu \mathrm{M})$ or gefitinib $(5 \mu \mathrm{M})$ were administered as single agents, or in combination, and cell viability was examined for $\leq 72 \mathrm{~h}$. While PC 3 cells displayed no beneficial cytotoxic effect with the combination of CPT and erlotinib, a trend of increased cytotoxicity with gefitinib was observed, however, it was not statistically significant (data not shown). On the contrary, the combination of either erlotinib or gefitinib with CPT increased the cytotoxic effect of CPT in MCF7 cells, compared to each treatment administered alone, as depicted in Fig. 1. After a 48-h treatment, erlotinib increased the anticancer effect of CPT from $24.3 \pm 8.2$ to $32.2 \pm 7.9 \%$ (a $7.9 \%$ difference; $\mathrm{p}<0.05$ ) at the lowest CPT dose examined $(0.02 \mu \mathrm{M}$ ), and from $42.9 \pm 10$ to $53.3 \pm 7.9 \%$ (a $10.4 \%$ difference) at $0.1 \mu \mathrm{M} \mathrm{CPT}$. Moreover, after 72-h treatment, erlotinib increased the anticancer effect of CPT from $30.7 \pm 5.8$ to $43.3 \pm 6.4 \%$ (a $12.7 \%$ difference; $\mathrm{p}<0.05$ ) at $0.02 \mu \mathrm{M}$ of CPT, and from $50.4 \pm 2.3$ to $64.2 \pm 1 \%$ (a $13.8 \%$ difference; $\mathrm{p}<0.05$ ) at $0.1 \mu \mathrm{M}$ of CPT. Similarly, the combination of gefitinib with CPT increased the cytotoxic effect of CPT, at $0.02 \mu \mathrm{M}$, from $30.4 \pm 4.3$ to $45 \pm 2.3 \%$ (a $14.6 \%$ difference; $\mathrm{p}<0.05$ ) after $48 \mathrm{~h}$, and from $46.3 \pm 5.1$ to $69.2 \pm 2 \%$ (a $22.9 \%$ difference; $p<0.05$ ) after $72 \mathrm{~h}$. However, no significant difference was observed with gefitinib at the highest dose of CPT $(0.1 \mu \mathrm{M})$. It is noteworthy that all combined treatments significantly $(\mathrm{p}<0.05)$ reduced the cytotoxic effect of erlotinib or gefitinib, as single agents, in MCF7 cells.

Combined treatment of gefitinib or erlotinib and CPT shows an increased inhibitory effect on topo I activity in PC3 and MCF7 cells. Previous data showed that certain tyrosine kinase antagonists, tyrphostins, can inhibit the activity of cellular topo I. In order to characterize the mechanism of the combined treatment, we sought to investigate the possibility that either erlotinib, or gefitinib, can exert a similar inhibitory effect. As the combined treatment demonstrated a significant cytotoxic effect after $48 \mathrm{~h}, \mathrm{MCF} 7$ and PC 3 cells were treated for $\leq 48 \mathrm{~h}$ with either 0.1 or $0.02 \mu \mathrm{M} \mathrm{CPT}$ and $1 \mu \mathrm{M}$ of erlotinib or gefitinib.

As depicted in Fig. 2, gefitinib reduced topo I activity in MCF7 cells by $28.6 \pm 12 \%(\mathrm{p}<0.05)$ after $24 \mathrm{~h}$ (Fig. $2 \mathrm{~A}$ and B); however, this effect was decreased after $48 \mathrm{~h}$ (not shown). CPT $(0.1 \mu \mathrm{M})$, as expected, reduced topo I activity by $47.1 \pm 7.3 \%$ $(\mathrm{p}<0.005)$. In cells treated with a combination of both drugs, a $62.8 \pm 1.1 \%$ decrease in topo I activity was observed, an additional decrease of $15.8 \pm 6.2 \%$ in topo I activity, compared to CPT alone $(\mathrm{p}<0.06)$ and a $34.3 \pm 10.9 \%$ additional decrease, compared to gefitinib alone $(\mathrm{p}<0.05)$, compatible with the increased cytotoxicity of both drugs (Fig. 1). When we examined the effect of gefitinib treatment on the PC3 cellular topo I activity (Fig. 2C and D) we observed a non-significant reduction $(11 \%)$ after $24 \mathrm{~h}$ (not shown), which increased after $48 \mathrm{~h}$ to $39.4 \pm 6.8 \%(\mathrm{p}<0.005)$. CPT $(0.1 \mu \mathrm{M})$ reduced topo I activity by $69.5 \pm 6.4 \%(\mathrm{p}<0.001)$. In cells treated with both 
A

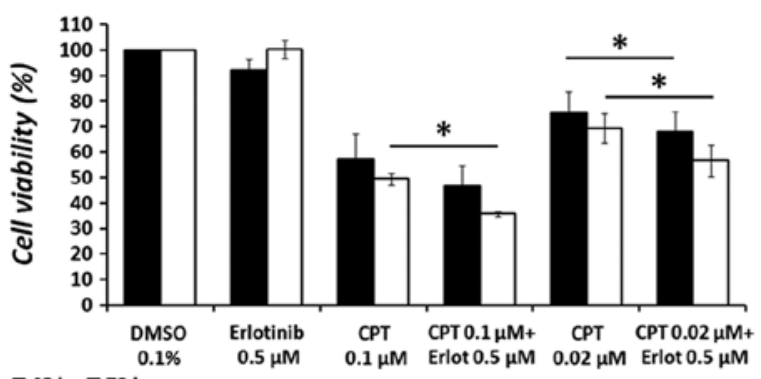

- $48 \mathrm{~h} \quad 072 \mathrm{~h}$

C

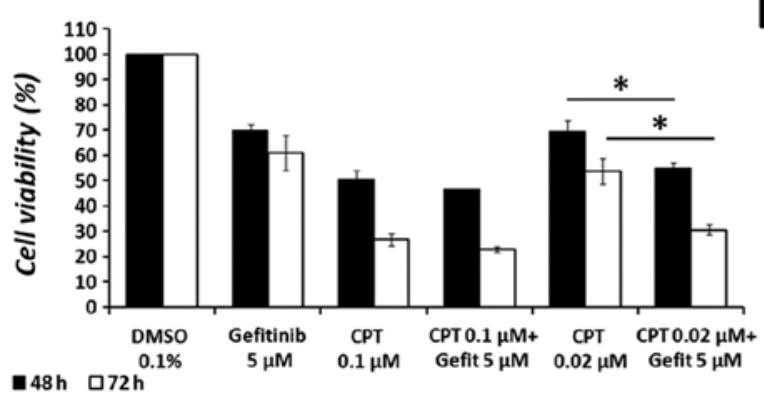

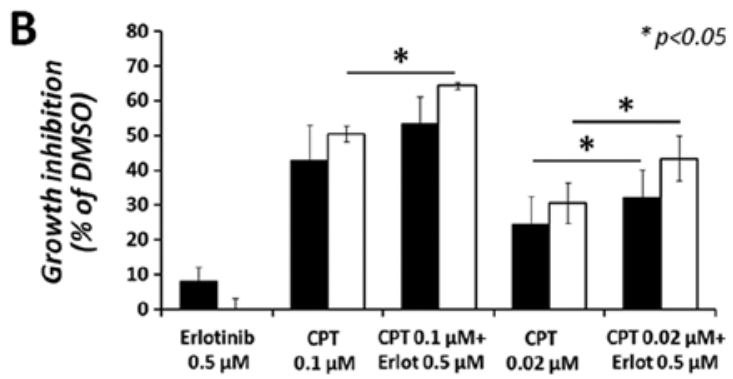

$48 \mathrm{~h} \quad 072 \mathrm{~h}$

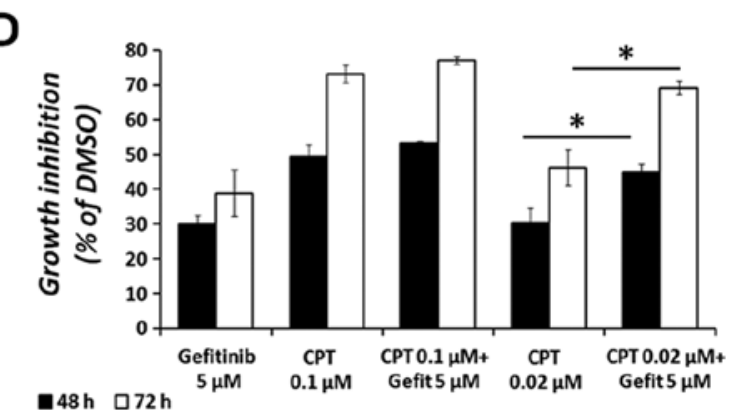

Figure 1. Effect of a combined CPT-erlotinib treatment on MCF7 cells. MCF7 cells were plated as triplicates on 96-well plates, at a density of 5,000 cells/ well and were allowed to adhere overnight. Cells were treated with CPT $(0.02$ or $0.1 \mu \mathrm{M})$ and $0.5 \mu \mathrm{M}$ erlotinib (A and B) or $5 \mu \mathrm{M}$ gefitinib $(\mathrm{C}$ and D), alone or in combination, for $\leq 72 \mathrm{~h}$. Cell viability was examined by the Neutral Red assay. Results represent mean \pm SE of 3 experiments. Statistical significance was determined by the Student's t-test analysis: "p $<0.05$.

A

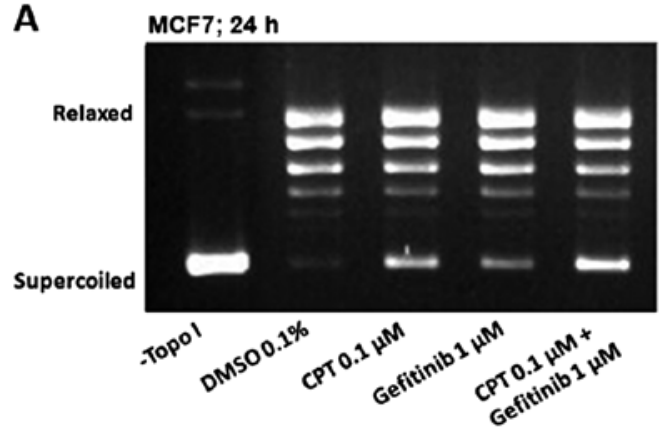

C

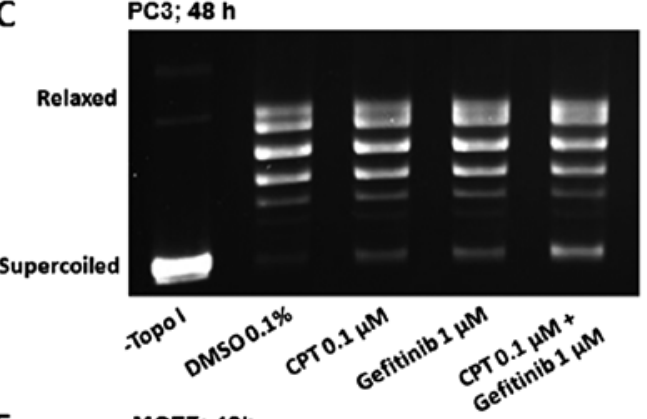

$\mathbf{E}$

MCF7; 48h

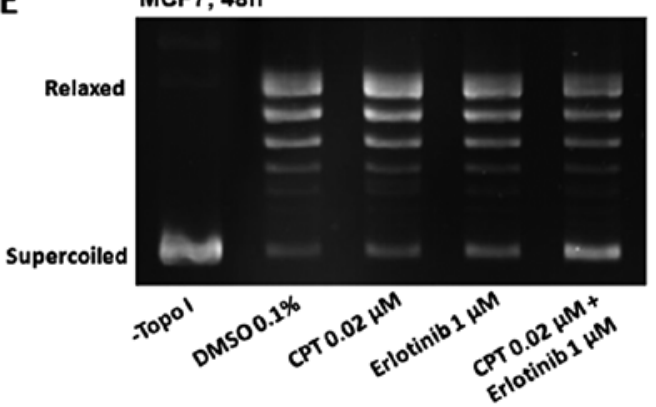

B
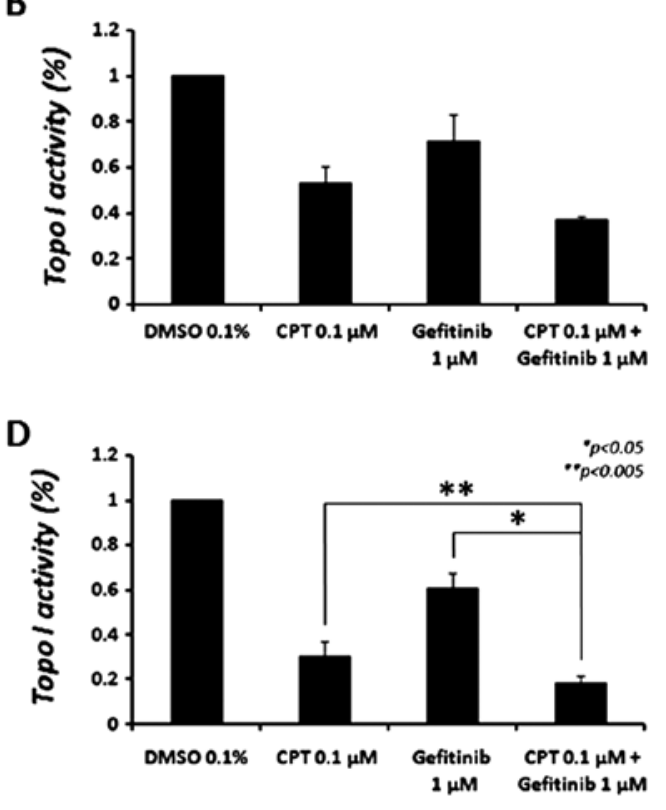

$\mathbf{F}$

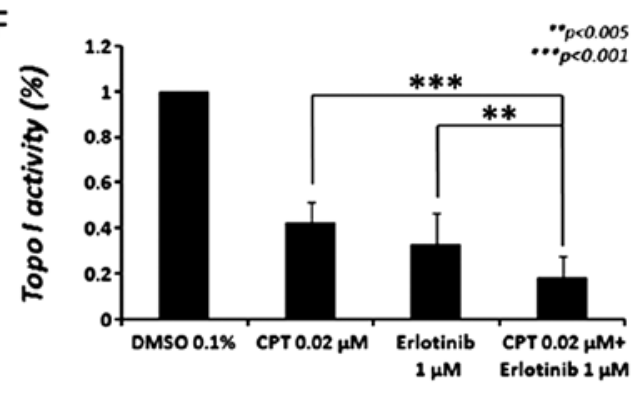

Figure 2. Combined effect of CPT and TKIs on cellular topo I. MCF7 or PC3 cells were treated with CPT (0.02 or $0.1 \mu \mathrm{M})$ and $1 \mu \mathrm{M}$ gefitinib (A-D) or erlotinib (E and F), alone or in combination, for $\leq 48 \mathrm{~h}$. Nuclear protein extracts were prepared and topo I activity was examined. Results represent mean \pm SE values of 3 experiments. Statistical significance was determined by the Student's t-test analysis: ${ }^{*} \mathrm{p}<0.05 ;{ }^{* *} \mathrm{p}<0.005 ;{ }^{* * *} \mathrm{p}<0.001$. 


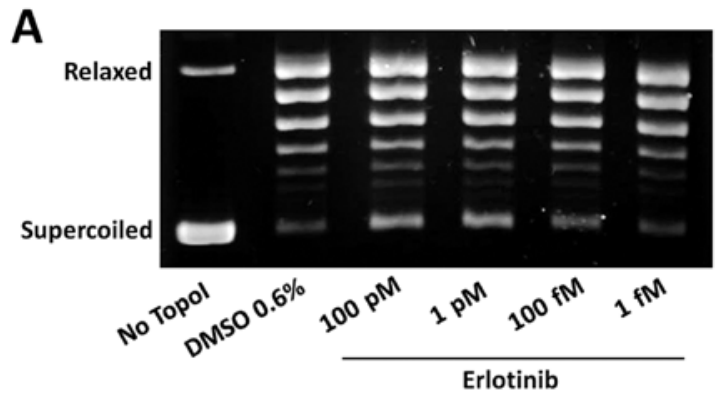

B

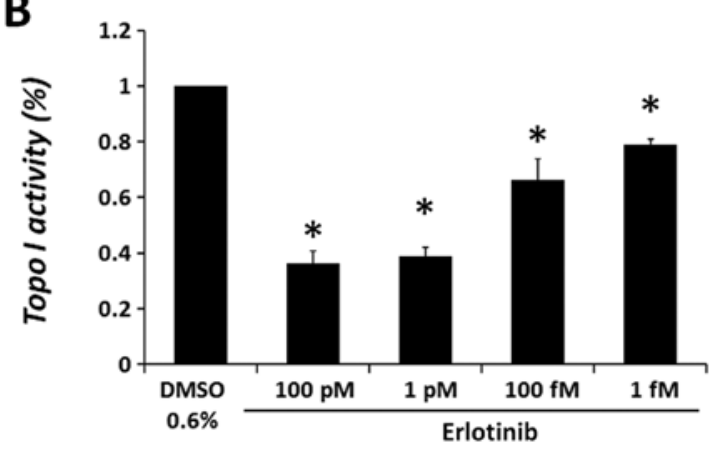

Figure 3. The effect of erlotinib on purified topo I activity. (A) Purified topo I activity. (B) Quantification analysis of purified topo I activity. One unit of commercial calf thymus topo I was added to a specific reaction mixture, containing different doses of erlotinib (100 pM-1 fM). Reaction products were analyzed by agarose gel electrophoresis. The results represent means $\pm \mathrm{SE}$ values of 3 experiments. Statistical significance was determined by the Student's t-test analysis: " $\mathrm{p}<0.05$, compared to the vehicle control (DMSO).

drugs, $81.7 \pm 3.3 \%$ decrease in topo I activity was observed, an additional decrease of $12.2 \pm 3.2 \%$ in topo I activity, compared to CPT alone $(\mathrm{p}<0.005)$ and $42.3 \pm 8.5 \%$ additional decrease, compared to gefitinib alone $(\mathrm{p}<0.05)$ was detected. Erlotinib reduced topo I activity in MCF7 cells by $66.8 \pm 7.8 \%(\mathrm{p}<0.005)$, $48 \mathrm{~h}$ after treatment (Fig. 2E and F); however, it did not reduce the enzyme activity in PC3 cells. As expected, CPT $(0.02 \mu \mathrm{M})$ reduced topo I activity by $57.3 \pm 5.3 \%(\mathrm{p}<0.001)$ in MCF7 cells after $48 \mathrm{~h}$. Similarly to gefitinib, when we examined the combined effect of these drugs on cellular topo I activity, an increased effect was observed $(81.6 \pm 5.4 \%$ reduction). This indicates that the combined treatment exhibited a $24.2 \pm 2.9 \%$ additional decrease in topo I activity compared to CPT alone $(\mathrm{p}<0.005)$ and a $14.7 \pm 2.6 \%$ additional decrease compared to erlotinib alone $(\mathrm{p}<0.001)$. These effects are compatible with the increased cytotoxicity of both drugs in MCF7 cells. While the results suggest that erlotinib and gefitinib, at the indicated doses, significantly reduce topo I activity, similarly to CPT, the different responses of MCF7 and PC3 cells to these drugs might be attributed to cell-specific properties.

Erlotinib, but not gefitinib, inhibits the DNA relaxation activity of purified topo I. As both gefitinib and erlotinib reduced topo I activity in the cell, we examined the mechanism underlying this inhibitory effect.

First, we investigated the direct effect of the drug on purified calf thymus topo I. One unit of the enzyme was added to a topo I reaction mixture in the presence of various concentrations of erlotinib or gefitinib and DNA relaxation activity was examined. As erlotinib exhibited reduced solubility at high concentrations, under topo I assay conditions, low doses of the drug were examined. The results depicted in Fig. 3 show that erlotinib significantly reduced the catalytic activity of purified topo I in a dose-dependent manner, compared to the vehicle control (DMSO). Erlotinib reduced topo I activity by $63.6 \pm 9.4 \%$, at the highest dose examined, $100 \mathrm{pM}$, while at the lowest dose of $1 \mathrm{fM}$, a $20.8 \pm 2.2 \%$ reduction in the enzyme activity was observed.

The addition of gefitinib to topo I reaction mixture did not affect the DNA relaxation activity of the enzyme, even at higher concentrations (data not shown), suggesting its indirect inhibitory effect on the cellular topo I.

Neither erlotinib nor gefitinib reduces the level of topo I protein. The reduction in topo I activity in erlotinib or gefitinib treated cells might be due to: i) reduction in the level of topo I protein, or ii) conformational changes, post-translational modifications or otherwise, in topo I protein that affect its activity. To examine the first possibility, cells were treated with erlotinib or gefitinib and CPT, as single agents or in combination, as indicated above. Nuclear extract proteins were analyzed by western blot analysis using specific anti-topo I antibody. The results depicted in Fig. 4 show that treatment of MCF7 or PC3 cells with either erlotinib or gefitinib alone did not alter the level of topo I protein. CPT treatment, as expected (27-29), reduced the level of free topo I protein. The combined treatments of CPT with erlotinib or gefitinib reduced topo I levels to a similar extent as CPT alone, suggesting that neither erlotinib nor gefitinib affect the level of topo I protein.

Erlotinib reduces the DNA-binding ability of topo I. The results obtained thus far suggest that treatment of cells with erlotinib might cause a transient inhibition of topo I activity, which alters the inhibitory effect of CPT. Our previous results demonstrated that other TKIs (certain tyrphostin derivatives) inhibited topo I activity by decreasing the ability of this enzyme to bind DNA in vitro (17). Therefore, to determine the effect of erlotinib on the DNA-binding ability of topo I, an EMSA assay was performed. A consensus 31-bp topo I DNA-binding sequence was used. Purified topo I was added to an enzyme-specific reaction mixture, which contained CPT $(120 \mu \mathrm{M})$ or erlotinib $(100 \mathrm{pM})$. High concentrations of the drugs are needed to establish a short time effect in vitro. The results depicted in Fig. 5 reveal a 36.2 $\pm 7.7 \%$ decrease $(\mathrm{p}<0.05)$ in the binding of topo I to the DNA, compared to the vehicle control (DMSO).

Erlotinib diminishes the CPT induced cleavable topo I-DNA complex. Since erlotinib reduced the DNA-binding ability of topo I in vitro, we sought to investigate whether this inhibitory activity is also exerted within the cell. In a transient reaction intermediate, termed the cleavable complex, topo I links covalently to DNA through a tyrosine residue in its active site, leaving a DNA break with a free 5'-hydroxyl end. Treatment of cells with CPT results in the stabilization of these cleavable complexes and the prevention of the DNA-ligation step (30-33). To examine the effect of erlotinib and the combined treatment with CPT on the formation of topo I-DNA cleavable complexes, we used the 'band depletion' assay (25). This assay is based on the observation that an 
A

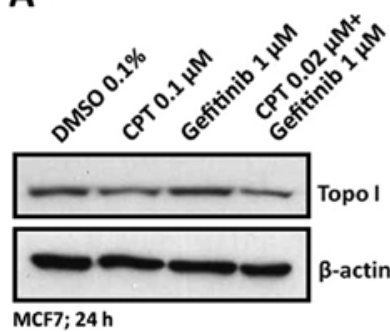

C

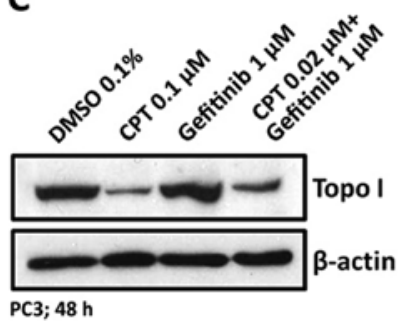

E

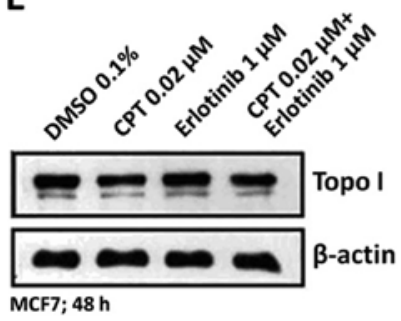

B

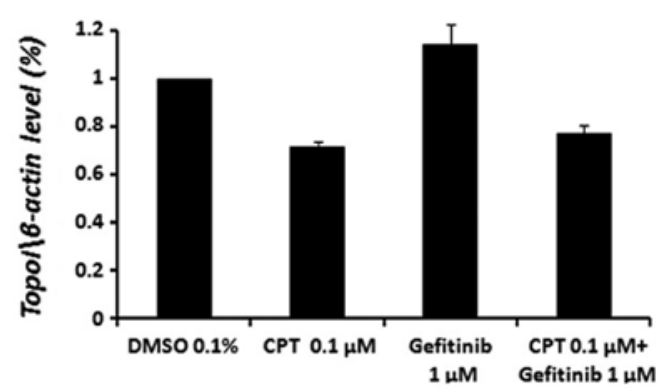

D

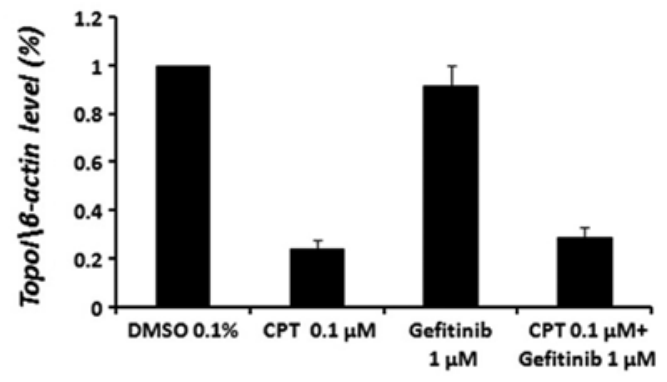

$\mathbf{F}$

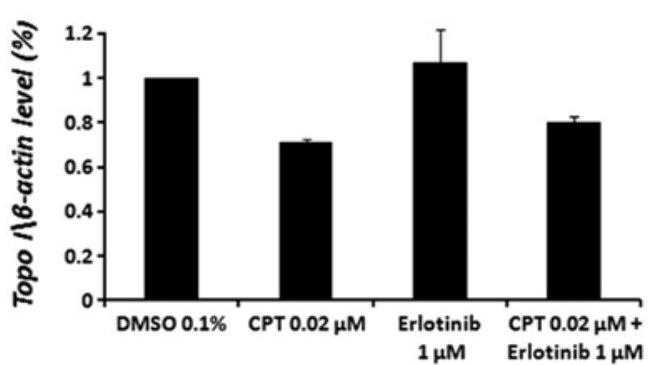

Figure 4. Topo I protein level in MCF7 and PC3 cells after CPT and TKI treatment. MCF7 or PC3 cells were treated with CPT (0.02 or $0.1 \mu \mathrm{M})$ and $1 \mu \mathrm{M}$ gefitinib (A-D) or erlotinib (E and F), alone or in combination, for 24 or $48 \mathrm{~h}$. Nuclear protein extracts were prepared and topo I protein level was determined by western blot analysis. Results represent mean \pm SE values of 3 experiments. Statistical significance was determined by the Student's t-test analysis: "p $<0.05$; ${ }^{* *} \mathrm{p}<0.001$, compared to the vehicle (DMSO) or the indicated TKI.
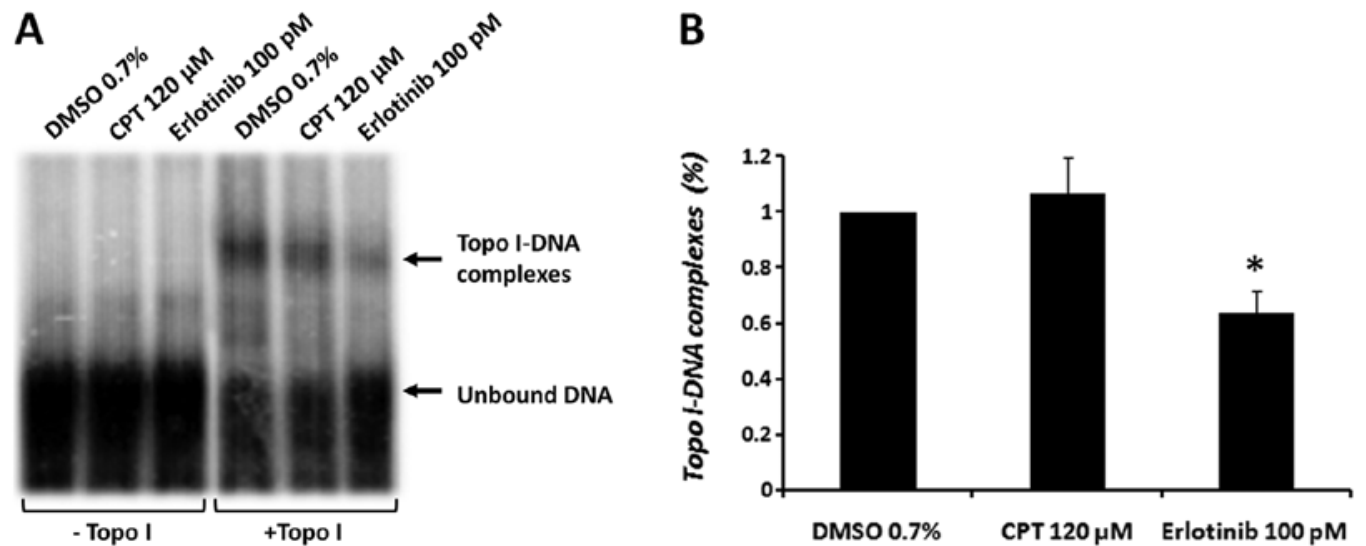

Figure 5. The effect of erlotinib and CPT on topo I-DNA-binding ability. (A) Purified calf-thymus topo I was added to a reaction mixture containing a ${ }^{32} \mathrm{P}$-labeled consensus topo I DNA-binding sequence in the presence of erlotinib (100 pM) or CPT (120 $\left.\mu \mathrm{M}\right)$. EMSA assay was performed. (B) Quantification analysis of topo I-DNA complexes. The results are means \pm SE values of 4 experiments. Statistical significance was determined by the Student's $t$-test analysis: * $\mathrm{p}<0.05$, compared to the vehicle (DMSO) or CPT.

increase in the amount of topo I bound to the DNA, because of CPT treatment, will cause depletion in the level of free topo I protein. Thus, the prevention of the enzyme ability to bind DNA will prevent the formation of topo I-DNA complexes by CPT, which will be manifested in band depletion reversal and increased level of free topo I. As this assay is used for shortterm treatments of the cells, high drug doses were employed. MCF7 cells were pre-treated with erlotinib for $3 \mathrm{~h}$, followed by CPT treatment for $1 \mathrm{~h}$, using similar drug concentrations $(30 \mu \mathrm{M})$. Cells were immediately lysed with SDS, and the level 
A

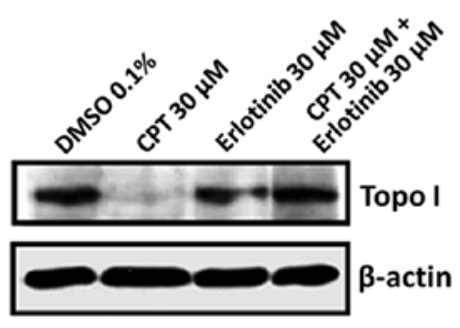

B

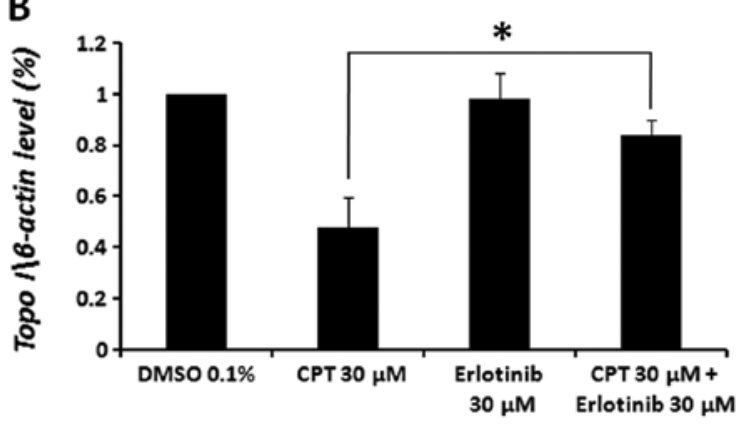

Figure 6. Erlotinib treatment reduces the level of CPT-induced DNA enzyme cleavable complexes. (A) MCF7 cells were treated with erlotinib (30 $\mu \mathrm{M}$ ) for $3 \mathrm{~h}$, followed by treatment with CPT $(30 \mu \mathrm{M})$ for $1 \mathrm{~h}$. Cells were immediately lysed by SDS, and equivalent volumes (48 $\mu \mathrm{l})$ were analyzed by western blot analysis. (B) Quantification analysis of topo I level relative to $\beta$-actin protein level. The results represent mean \pm SE values of 3 experiments. Statistical significance was determined by the Student's t-test analysis: * $\mathrm{p}<0.05$.

of topo I protein was examined by western blot analysis, using specific anti-topo I antibodies. The results depicted in Fig. 6 demonstrate a significant decrease $(51.7 \pm 12 \%$ compared to the vehicle control) in the level of free topo I in CPT-treated cells, and no effect on the level of topo I in cells treated with erlotinib alone. Pretreatment with erlotinib, before administering CPT, abolished the CPT-induced cleavable complexes and restored the level of topo I enzyme to $84.2 \pm 6.1 \%$.

\section{Discussion}

Preclinical and clinical studies have demonstrated the antitumor activity of EGFR-TK inhibitors, as single agents or in combination with other drugs, including topoisomerase interacting agents (8-11). It has been shown in vitro and in vivo, that the combined treatment of human colorectal cancer cells, with anti-EGFR drugs (cetuximab or gefitinib) and with topoisomerase I inhibitor topotecan (TPT), increased the antitumor activity of either agent alone $(8,9,19)$. Phase II studies have demonstrated that combined therapy of triple negative breast cancer (TNBC) with cetuximab (an anti-EGFR antibody) and cisplatin dramatically increased the patient response rate, compared to cisplatin treatment alone, and doubled their progression-free survival duration $(20,34)$. Indeed, cisplatin has been shown to inhibit the activity and level of topoisomerases $(35,36)$. Gefitinib was found to modulate the ability of SN-38 (the active metabolite of CPT-11) to inhibit topo I in colorectal tumor cell lines, to accumulate cells in S-phase, and to induce apoptosis (21). Erlotinib was found to inhibit tumor growth and metastasis in a TNBC xenograft model (20) and was shown to increase the antitumor activity of CPT-11 in colorectal xenografts, with no increased toxicity (10).

We previously showed that certain tyrosine kinase antagonists, tyrphostins, inhibit the catalytic activity of the cellular topoisomerase I (topo I) $(17,18)$. Since the activity of TKIs is not correlated with EGFR expression $(10,15)$, and excluding EGFR mutations, resistance mechanisms have been linked to several cellular processes, such as activation of alternative tyrosine kinase receptors (such as IGF-1R) (37), constitutive ligand-independent activation of ERK or Akt $(10,37)$, and expression of MDR transporter proteins $(38,39)$, we examined the mechanism of the combined treatment with topoisomerases- and EGFR-targeting agents by investigating the possibility that erlotinib and gefitinib, which are currently used in chemotherapy treatments (unlike tyrphostins), exert their anticancer activity by also affecting topoisomerase I.

MCF7 breast cancer and PC3 prostate cancer cell lines were used to examine the combined effect of topo I and EGFR-TK inhibitors, and as the source for the cellular topo I. While the combined treatments with CPT, a known topo I inhibitor, and neither erlotinib nor gefitinib exhibit any significant beneficial effects in PC3 cells, an increased cytotoxicity was observed in MCF7 cells. To investigate the effect of the combined treatment on topo I, cells were treated with low concentrations of the indicated drugs to avoid cell toxicity. Interestingly, gefitinib was shown to reduce the cellular topo I activity in both MCF7 and PC3 cells, while erlotinib reduced cellular topo I activity in MCF7 cells only. These differences in cellular response suggest that both erlotinib and gefitinib exhibit a cell type dependent inhibition of topo I and the determination of the combined treatment depends on the cell type and on the assay conditions. Furthermore, in compatibility with the increased cell cytotoxicity, the combined treatment increased the inhibitory effect on topo I activity, compared to that observed by each of the drugs administered alone.

Examination of the mechanism by which topo I activity is reduced by either gefitinib or erlotinib, was performed using various parameters. The reduction of topo I activity in treated cells was not due to a reduction in the enzyme protein level, suggesting possible modifications of the enzyme protein that cause this inhibitory effect. First we examined a possible direct interaction between the drugs and a purified topo I enzyme and found that only erlotinib reduced the DNA relaxation activity of the topo I, in a dose-dependent manner, while gefitinib did not affect the enzyme activity. The ability of erlotinib to directly inhibit topo I at very low doses, points to its potential as a potent topo I inhibitor. It is not yet clear why higher doses of this drug exerted less inhibitory effect in vitro, however, we found that erlotinib, at higher concentration, forms microaggregates under our assay conditions, which might interfere with its binding to the target and may diminish its inhibitory ability (data not shown). The reduction of topo I activity by erlotinib, in vitro, is due to modifications of the DNA binding ability of the enzyme, as observed by the EMSA experiment. Since no effect on the mobility of the DNA was observed, it is probable that erlotinib, by itself, did not bind to the DNA. 
Therefore, it is possible that erlotinib affects the conformation of topo I protein by a direct interaction with the enzyme, in a way that changes its DNA binding activity. Since we previously showed that tyrphostins, which are tyrosine kinase inhibitors, act also as topo I antagonists by altering the conformation of topo I and inhibiting its DNA binding ability $(17,18)$, our present data strengthen the notion that certain TKIs exert their inhibitory activity by interacting with topo I protein.

As the in vitro data suggest that erlotinib interferes with the DNA binding ability of topo I enzyme, it is possible that this is also its mode of action in the cell. To examine this notion, we utilized the mode of action of CPT, which stabilized the DNA-enzyme cleavable complexes, and showed that pretreatment of the cells with erlotinib, prior to CPT treatment, diminished the formation of CPT induced DNA-enzyme cleavable complexes. This result suggests that pretreatment of the cells with erlotinib modified the ability of topo I to bind to the DNA and therefore less DNA-enzyme cleavable complexes were stabilized in the presence of CPT. These data are compatible with the in vitro results and with our previous finding with other tyrosine kinase inhibitors $(17,18)$.

Alternatively, it is known that the activity of topo I in the cell is regulated by phosphorylation of the serine/threonine or tyrosine residues of the enzyme protein $(1,31,32)$. EGFR signal transduction pathway involves activation of serine/threonine kinases such as PKC (40). PKC and casein kinase II were shown to phosphorylate topo I and thus regulate its activity (31). Therefore, it is possible to suggest that the inhibition of EGFR signal transduction pathway by erlotinib will also affect PKC activity and reduce the phosphorylation level of topo I, thereby reducing its activity, as previously suggested (31). This is supported by the inhibition of cellular topo I in gefitinbtreated cells, which exhibits a different topo I-inhibitory mechanism than that of erlotinib. Gefitinib was not found to directly interact with topo I enzyme in vitro, however, it was found to modulate EGFR signaling pathways (data not shown). As topo I protein level is not reduced after gefitinib treatment, the reduction in the enzyme activity can be a cause of a postranslational modification, such as hypo-phosphorylation, that might be mediated by the blocking of EGFR signaling. Indeed, it has been suggested that gefitinib can induce inhibition of PKC pathway (41).

Topo I inhibition could also be dependent on the inhibition of other cellular proteins. It has been shown that erlotinib treatment suppresses the activity of certain cyclin-dependent kinases (CDKs) in breast cancer (42), inhibits the activity of a mutant JAK2, that is found in polycythemia vera (PV) and other proliferative disorders (43). Gefitinib was shown to inhibit and influence the production of certain growth factors and cytokines, such as VEGF, bFGF and TGF- $\alpha$ (15). Several studies have shown that both gefitinib and erlotinib can modulate the activity of ABC-transporters (44-47) and inhibit the ErbB-2 receptor $(15,48)$, thus one may assume that other proteins might be affected by these drugs as well, including topoisomerases.

The results of this study suggest that topo I is a novel target of erlotinib, in addition to its known activity as PTK inhibitor. Therefore, simultaneous inhibition of essential cellular enzymes, such as topo I and protein tyrosine kinases, may serve as potent anticancer strategy. Our results point also to the possibility that a combination of erlotinib and gefitinib with topo I inhibitors may demonstrate an effective anti-breast cancer treatment.

\section{Acknowledgements}

This study was supported in part by the Ben-Gurion University Seed Research fund.

\section{References}

1. Cretaio E, Pattarello L, Fontebasso Y, Benedetti P and Losasso C: Human DNA topoisomerase IB: structure and functions. Ital J Biochem 56: 91-102, 2007.

2. Forterre P, Gribaldo S, Gadelle D and Serre M: Origin and evolution of DNA topoisomerases. Biochimie 89: 427-446, 2007.

3. Wang JC: DNA topoisomerases. Annu Rev Biochem 65: 635-692, 1996.

4. Corbett KD and Berger JM: Structure, molecular mechanisms, and evolutionary relationships in DNA topoisomerases. Annu Rev Biophys Biomol Struct 33: 95-118, 2004.

5. Tomicic MT and Kaina B: Topoisomerase degradation, DSB repair, p53 and IAPs in cancer cell resistance to camptothecinlike topoisomerase I inhibitors. Biochim Biophysica Acta 1835: 11-27, 2013

6. Haglof KJ, Popa E and Hochster HS: Recent developments in the clinical activity of topoisomerase-1 inhibitors. Cancer Ther 1: 117-145, 2006.

7. Pommier Y: Topoisomerase I inhibitors: camptothecins and beyond. Nat Rev Cancer 6: 789-802, 2006.

8. Ciardiello F, Caputo R, Bianco R, et al: Antitumor effect and potentiation of cytotoxic drugs activity in human cancer cells by ZD-1839 (Iressa), an epidermal growth factor receptor-selective tyrosine kinase inhibitor. Clin Cancer Res 6: 2053-2063, 2000.

9. Koizumi F, Kanzawa F, Ueda Y, et al: Synergistic interaction between the EGFR tyrosine kinase inhibitor gefitinib ('Iressa') and the DNA topoisomerase I inhibitor CPT-11 (irinotecan) in human colorectal cancer cells. Int J Cancer 108: 464-472, 2004.

10. Chen J, Smith M, Kolinsky K, et al: Antitumor activity of HER1/ EGFR tyrosine kinase inhibitor erlotinib, alone and in combination with CPT-11 (irinotecan) in human colorectal cancer xenograft models. Cancer Chemother Pharmacol 59: 651-659, 2007.

11. Friedmann B, Caplin M, Hartley JA and Hochhauser D: Modulation of DNA repair in vitro after treatment with chemotherapeutic agents by the epidermal growth factor receptor inhibitor gefitinib (ZD1839). Clin Cancer Res 10: 6476-6486, 2004.

12. Normanno N, Bianco C, De Luca A, Maiello MR and Salomon DS: Target-based agents against ErbB receptors and their ligands: a novel approach to cancer treatment. Endocr Relat Cancer 10: 1-21, 2003.

13. Uberall I, Kolár Z, Trojanec R, Berkovcová J and Hajdúch M: The status and role of ErbB receptors in human cancer. Exp Mol Pathol 84: 79-89, 2008.

14. Oda K, Matsuoka Y, Funahashi A and Kitano H: A comprehensive pathway map of epidermal growth factor receptor signaling. Mol Syst Biol 1: 2005.0010, 2005.

15. Harari PM: Epidermal growth factor receptor inhibition strategies in oncology. Endocr Relat Cancer 11: 689-708, 2004.

16. Smith J: Erlotinib: small-molecule targeted therapy in the treatment of non-small-cell lung cancer. Clin Ther 27: 1513-1534, 2005.

17. Aflalo E, Seri I, Segal S, Gazit A and Priel E: Inhibition of topoisomerase I activity by tyrphostin derivatives, protein tyrosine kinase blockers: mechanism of action. Cancer Res 54: 5138-5142, 1994.

18. Bendetz-Nezer S, Gazit A and Priel E: DNA topoisomerase I as one of the cellular targets of certain tyrphostin derivatives. Mol Pharmacol 66: 627-634, 2004.

19. Ciardiello F, Bianco R, Damiano V, et al: Antitumor activity of sequential treatment with topotecan and anti-epidermal growth factor receptor monoclonal antibody C225. Clin Cancer Res 5: 909-916, 1999.

20. Ueno NT and Zhang D: Targeting EGFR in triple negative breast cancer. J Cancer 2: 324-328, 2011. 
21. Azzariti A,XuJ,PorcelliLandParadiso A: The schedule-dependent enhanced cytotoxic activity of 7-ethyl-10-hydroxy-camptothecin (SN-38) in combination with Gefitinib (Iressa, ZD1839). Biochem Pharmacol 68: 135-144, 2004.

22. Johnston MD, Finter NB and Young PA: Dye uptake method for assay of interferon activity. Methods Enzymol 78: 394-399, 1981.

23. Auer B, Vosberg HP, Buhre U, Klocker H, Hirsch-Kauffmann M and Schweiger M: Intracellular distribution of DNA topoisomerase I in fibroblasts from patients with Fanconi's anaemia. Hum Genet 61: 369-371, 1982.

24. Sambrook J, Fritsch EF and Maniatis T: Molecular Cloning: A Laboratory Manual. Cold Spring Harbor Laboratory Press, Cold Spring Harbor, NY, 1989.

25. Kaufmann SH and Svingen PA: Immunoblot analysis and band depletion assays. In: Methods in Molecular Biology, DNA topoisomerase protocols: DNA Topoisomerase Protocols. Bjornsti MA and Osheroff N (eds). Vol. 94. Humana Press Inc., Totowa, NJ: 253-268, 1999.

26. Stevnsner T, Mortensen UH, Westergaard O and Bonven BJ: Interaction between eukaryotic DNA topoisomerase I and a specific binding sequence. J Biol Chem 264: 10110-10113, 1989.

27. Desai SD, Li TK, Rodriguez-Bauman A, Rubin EH and Liu LF: Ubiquitin/26S proteasome-mediated degradation of topoisomerase I as a resistance mechanism to camptothecin in tumor cells. Cancer Res 61: 5926-5932, 2001.

28. Desai SD, Liu LF, Vazquez-Abad D and D'Arpa P: Ubiquitindependent destruction of topoisomerase I is stimulated by the antitumor drug camptothecin. J Biol Chem 272: 24159-24164, 1997.

29. Desai SD, Zhang H, Rodriguez-Bauman A, et al: Transcriptiondependent degradation of topoisomerase I-DNA covalent complexes. Mol Cell Biol 23: 2341-2350, 2003.

30. Wang HK, Morris-Natschke SL and Lee KH: Recent advances in discovery and development of topoisomerase inhibitors as antitumor agents. Med Res Rev 17: 367-425, 1997.

31. Pommier Y, Pourquier P, Fan Y and Strumberg D: Mechanism of action of eukaryotic DNA topoisomerase I and drugs targeted to the enzyme. Biochim Biophys Acta 1400: 83-105, 1998.

32. Wang JC: Cellular roles of DNA topoisomerases: a molecular perspective. Nat Rev Mol Cell Biol 3: 430-440, 2002.

33. Wang JC: DNA topoisomerases as targets of therapeutics: an overview. Adv Pharmacol 29A: 1-19, 1994.

34. Baselga J, Gomez P, Awada A, et al: The addition of cetuximab to cisplatin increases overall response rate (ORR) and progressionfree survival (PFS) in metastatic triple-negative breast cancer (TNBC): results of a randomized phase II study (BALI-1). Ann Oncol 21 (Suppl 8): 2740, 2010.

35. Wu X, Yalowich JC and Hasinoff BB: Cisplatin inhibits the catalytic activity of DNA topoisomerase II by binding to critical protein thiol groups and by binding to DNA. AACR Meeting: Experimental and Molecular Therapeutics 31: Topoisomerase, Telomerase, and Nucleosides/Nucleotides. Abst. 3079, 2004.
36. Aoe K, Kiura K, Ueoka H, et al: Cisplatin down-regulates topoisomerase I activity in lung cancer cell lines. Anticancer Res 24 3893-3897, 2004

37. Camp ER, Summy J, Bauer TW, Liu W, Gallick GE and Ellis LM: Molecular mechanisms of resistance to therapies targeting the epidermal growth factor receptor. Clin Cancer Res 11: 397-405, 2005.

38. Chen YJ: Mechanisms underlying resistance to epidermal growth factor receptor inhibitors in non-small cell lung cancer. Biol Biomed Rep 2: 141-148, 2012

39. Chen YJ, Huang WC, Wei YL, et al: Elevated BCRP/ABCG2 expression confers acquired resistance to gefitinib in wild-type EGFR-expressing cells. PLoS One 6: e21428, 2011.

40. Jorissen RN, Walker F, Pouliot N, Garrett TP, Ward CW and Burgess AW: Epidermal growth factor receptor: mechanisms of activation and signalling. Exp Cell Res 284: 31-53, 2003.

41. Kim H, Kim SH, Kim MJ, et al: EGFR inhibitors enhanced the susceptibility to NK cell-mediated lysis of lung cancer cells. J Immunother 34: 372-381, 2011.

42. Ling YH, Li T, Yuan Z, Haigentz M Jr, Weber TK and PerezSoler R: Erlotinib, an effective epidermal growth factor receptor tyrosine kinase inhibitor, induces $\mathrm{p} 27^{\mathrm{KIP1}}$ up-regulation and nuclear translocation in association with cell growth inhibition and G1/S phase arrest in human non-small-cell lung cancer cell lines. Mol Pharmacol 72: 248-258, 2007.

43. Li Z, Xu M, Xing S, et al: Erlotinib effectively inhibits JAK2 $2^{\mathrm{V} 617 \mathrm{~F}}$ activity and polycythemia vera cell growth. J Biol Chem 282 3428-3432, 2007

44 Shi Z, Peng XX, Kim IW, et al: Erlotinib (Tarceva, OSI-774) antagonizes ATP-binding cassette subfamily B member 1 and ATP-binding cassette subfamily $\mathrm{G}$ member 2-mediated drug resistance. Cancer Res 67: 11012-11020, 2007.

45. Yang CH, Huang CJ, Yang CS, et al: Gefitinib reverses chemotherapy resistance in gefitinib-insensitive multidrug resistant cancer cells expressing ATP-binding cassette family protein. Cancer Res 65: 6943-6949, 2005.

46. Yanase K, Tsukahara S, Asada S, Ishikawa E, Imai Y and Sugimoto Y: Gefitinib reverses breast cancer resistance protein-mediated drug resistance. Mol Cancer Ther 3: 1119. $1125,2004$.

47. Marchetti S, de Vries NA, Buckle T, et al: Effect of the ATP-binding cassette drug transporters ABCB1, ABCG2, and ABCC2 on erlotinib hydrochloride (Tarceva) disposition in in vitro and in vivo pharmacokinetic studies employing Bcrp1 ${ }^{-1-}$ Mdrla/ $/ \mathrm{b}^{-/}$(triple-knockout) and wild-type mice. Mol Cancer Ther 7: 2280-2287, 2008

48. Normanno N, Maiello MR and De Luca A: Epidermal growth factor receptor tyrosine kinase inhibitors (EGFR-TKIs): simple drugs with a complex mechanism of action? J Cell Physiol 194 13-19, 2002. 